\title{
Thermal Energy Communication By Unsteady Mhd Run Precedent A Perpendicular Absorbent Plate Captivated In A Absorbent Average
}

\author{
P. Sreedivya, Dr. K. Sunitha
}

\begin{abstract}
In the present paper learn thermal energy communication by unsteady MHD Run Precedent a perpendicular absorbent laminate captivated in a absorbent average.. a moment needy suction to the plate into a time dependent, parameter and the corresponding momentum and energy equations have been solved numerically the non linear governing partial differential equation obtained are converted into PDE.non dimensional rate and high temperature profiles be next in presence graphically for different value of the parameter entering into the problem .
\end{abstract}

Keywords: Thermal radiation, MHD, Unsteady, Suction, Porous medium

\section{Introduction}

The free convection going on the accelerate flow of a viscous incompressible liquid precedent an endless perpendicular absorbent plate with suction has many significant technological applications in the astrophysical, geophysical and engineering problems. The heat of rooms and buildings by the use of radiators is a recognizable .high temperature transport by free of charge convection to Heat losses from hot pipes, ovens etc surrounded by cooler air, are at least in part, due to free convection.. This is due to the significant role of thermal radiation in exterior temperature transport while convection heat relocate is similar, particularly in free convection problems involving absorbing emitting fluids. In the present article we examine the thermal emission communication on an absorbing emitting fluid permitted by a transversely applied magnetic field past a moving vertical porous plate embedded in a porous medium with time dependent suction and temperature. The similarity solutions are then obtained numerically for various parameters entering into the problem and discussed them from the physical point of view.

\section{Review Of Literature}

1. Soundalgekar and Takhar (1981) Radiation belongings on free convection flow of a gas past a half unlimited horizontal plate.

2. Hossain and Takhar (1996):The outcome of radiation using the Rosseland diffusion estimate on diverse convection the length of a perpendicular plate with uniform free stream velocity and outside high temperature.

3. Ali et al. (1984) radiation outcome on normal convection flow more than a perpendicular outside in a ancient gas.

4. Mansour (1990): the communication of mixed convection with thermal emission in laminar border layer flow over a straight, continuous moving sheet with suction/injection.

5. Whereas Albraba et al. (1992) studied the same problem considering magnetic effect taking into account the binary chemical response and Soret - Doufour effects.

6. Seigel (1958): passing free convection flow precedent a half endless perpendicular plate by an integral method.

7. Yamamoto et al. (1976) investigated the acceleration of convection in a absorbent permeable medium along an arbitrary but smooth surface.

8. Raptis (1983) :free convection in a absorbent average surrounded by an endless plate. Raptis and Perdikis (1985) without charge convection flow through a porous medium bounded by a half endless perpendicular absorbent plate.

9. Singh and Dikshit (1988). Sattar et al. (2000) trembling free convection flow the length of a perpendicular absorbent plate surrounded in a absorbent medium.

10. Alam and Rahman (2005): MHD free convection flow and mass transfer along a perpendicular absorbent cover in a absorbent medium allowing for Soret-Dofour effects.

11. Sattar and Kalim (1996) studied the effects of unsteady free convection interaction with thermal radiation in a boundary layer flow.

12. El-Arabawy (2003) consequence of suction/injection on a micro Antarctic fluid precedent a endlessly moving with radiation. 
13. Ferdows et al. (2004) examine the thermal radiation communication by convection in a boundary layer stream at a perpendicular cover with variable suction

\section{Mathematical Formulation}

Let us consider the problem of a trembling MHD free convection flow of a thick, incompressible and electrically conduct liquid along a perpendicular absorbent flat plate under the pressure of a consistent maganatic field

1. The flow is implicit to be in the $\mathrm{x}$-direction, which is in use the length of the plate in the upward direction

2. $y$-axis normal to the plate. originally it is understood so as to the cover and the fluid be at a steady high temperature $\mathrm{T}_{\infty}$ at every one points.

3. on time $t>0$ the plate is implicit to be affecting in the increasing way with the velocity $U(t)$ and there is a suction velocity $\mathrm{v}_{0}(\mathrm{t})$ in use to be a function of time, the high temperature of the plate raise to $\mathrm{T}(\mathrm{t})$ where $\mathrm{T}(\mathrm{t})>\mathrm{T}_{\infty}$.

4. The plate is measured to be of endless length, all derivatives with respect to $\mathrm{x}$ vanish and so the physical variables are functions of $y$ and $t$ only.

V.The fluid is measured to be gray; absorbing-emitting radiation but non-scattering medium and the Roseland approximation is used to describe the radioactive high temperature instability in the $\mathrm{x}$-direction is considered negligible in assessment to the y-direction.

vi.Assuming that the Boussinesq and boundary-layer approximation grip and using the Darcy-Forchhemier model, the governing equations for the problem are as follows:

Continuity equation

$$
\frac{\partial v^{\prime}}{\partial y^{\prime}}=0
$$

Momentum equation

$$
\frac{\partial u^{\prime}}{\partial t^{\prime}}+v^{\prime} \frac{\partial u^{\prime}}{\partial y^{\prime}}=g \beta\left(T-T_{\infty}\right)+g \beta^{*}\left(C-C_{\infty}\right)+v^{\prime} \frac{\partial^{2} u^{\prime}}{\partial y^{\prime 2}}-\sigma \frac{B_{0}^{2}}{\rho} u^{\prime}-v \frac{u^{\prime}}{K^{*}}
$$

Energy equation

$$
\begin{gathered}
\frac{\partial T}{\partial t^{\prime}}+v^{\prime} \frac{\partial T}{\partial y^{\prime}}=\alpha \frac{\partial^{2} T}{\partial y^{\prime 2}}-\frac{1}{\rho c_{p}} \frac{\partial q_{r}}{\partial y^{\prime}}-\frac{v}{c_{p}}\left(\frac{\partial u^{\prime}}{\partial y^{\prime}}\right)^{2} \\
\frac{\partial C}{\partial t^{\prime}}+v^{\prime} \frac{\partial C}{\partial y^{\prime}}=D \frac{\partial^{2} C}{\partial y^{\prime 2}}-K_{r}^{2}\left(C-C_{\infty}\right)
\end{gathered}
$$

where $(\mathrm{u}, \mathrm{v})$ are the components of velocity along the $\mathrm{x}$-and $\mathrm{y}$-directions respectively

$\mathrm{t}=$ time,

$v=$ kinematic viscosity,

$\rho=$ density of the fluid,

$\mathrm{g}_{0}=$ acceleration due to gravity,

$\beta=$ coefficient of volume expansion,

$\mathrm{B}_{0}=$ magnetic induction,

$\mathrm{T}$ and $\mathrm{T}_{\infty}=$ temperature of the fluid within the boundary layer and in the free stream respectively,

$\sigma=$ electric conductivity,

$\alpha=$ thermal diffusivity

$\mathrm{c}_{\mathrm{p}}=$ specific heat at constant pressure,

$\mathrm{k}=$ permeability of the porous medium.

The comparable boundary circumstances for the over difficulty are given by By using Rosseland approximation $\mathrm{q}_{\mathrm{r}}$ takes the form

$$
\begin{aligned}
& u^{\prime}=U_{0}, T=T_{w}+\varepsilon\left(T_{w}-T_{\infty}\right) e^{n^{\prime} t^{\prime}}, \\
& C=C_{w}+\varepsilon\left(C_{w}-C_{\infty}\right) e^{n^{\prime} t^{\prime}} \text { at } y^{\prime}=0 \\
& u^{\prime} \rightarrow U^{*}\left(t^{\prime}\right), \mathrm{T} \rightarrow \mathrm{T}_{\infty}, \mathrm{C} \rightarrow \mathrm{C}_{\infty}, \text { at } \mathrm{y}^{\prime} \rightarrow \infty
\end{aligned}
$$

where $\sigma_{1}$, the Stefan-Boltzamann constant and $\mathrm{k}_{1}$, the mean absorption coefficient. It is assumed that the temperature differences within the flow are satisfactorily small such that $\mathrm{T}^{4}$ may be articulated as a linear meaning of high temperature. This is consummate by expanding $\mathrm{T}^{4}$ in a Taylor series about $\mathrm{T}_{\infty}$ and neglecting higher-order terms, thus 


$$
q_{r}=-\frac{4 \sigma_{s}}{3 k_{e}} \frac{\partial T^{4}}{\partial y^{\prime}}
$$

Using (5) and (6) in equation (3) we have

$$
T^{4} \cong 4 T_{0}^{3} T-3 T_{\infty}^{4}
$$

In order to obtain a similarity solution in time of the problem, we introduce a similarity parameter $\delta$ as $\delta=\delta(t)$,

$$
\frac{\partial \mathrm{T}}{\partial t^{\prime}}+v^{\prime} \frac{\partial \mathrm{T}}{\partial y^{\prime}}=\alpha \frac{\partial^{2} \mathrm{~T}}{\partial y^{\prime 2}}+\frac{16 \sigma_{s} T_{\infty}^{3}}{3 k_{e} \sigma c_{p}} \frac{\partial^{2} \mathrm{~T}}{\partial y^{\prime 2}}+\frac{v}{c_{p}}\left(\frac{\partial \mathrm{u}^{\prime}}{\partial y^{\prime}}\right)
$$

such that $\delta$ is a length scale.

With this similarity parameter, a similarity variable is then introduced as

$$
v^{\prime}=-V_{0}\left(1+\varepsilon e^{n^{\prime} t^{\prime}}\right)
$$

In terms of this length scale, a suitable solution of the equation (1) can be taken as where $\mathrm{v}_{0}$ is the mass transfer parameter, which is +ve for suction and -ve for injection.

Following Sattar and Hossain (1992) U(t) and T(t) are now consider to have the following form:

$$
\begin{aligned}
& y=\frac{y^{\prime} V_{0}}{v}, t=\frac{t^{\prime} V_{0}^{2}}{v}, u=\frac{u^{\prime}}{U_{0}}, V=\frac{v}{V_{0}}, u(t)=\frac{U^{*}\left(t^{\prime}\right)}{U_{0}}, \\
& n=\frac{n^{\prime} V}{V_{0}^{2}}, G_{r}=\frac{v g \beta\left(T_{w}-T_{\infty}\right)}{U_{0} V_{0}^{2}}, G_{m}=\frac{v g \beta^{*}\left(C_{w}-C_{\infty}\right)}{U_{0} V_{0}^{2}}, \\
& M=\frac{\sigma \beta_{0}^{2} v}{U_{0}^{2}}, R=\frac{16 \sigma_{s} T_{\infty}^{3}}{e k_{e} k}, \theta=\frac{T-T_{\infty}}{T_{w}-T_{\infty}}, \\
& \varphi=\frac{C-C_{\infty}}{C_{w}-C_{\infty}}, P_{r}=\frac{v}{\alpha}, S_{c} \frac{v}{D}, E_{c}=\frac{U_{0}^{2}}{c_{p}\left(T_{w}-T_{\infty}\right)}, \\
& K=\frac{K^{*} V_{0}^{2}}{v^{2}}, K_{r}^{2}=\frac{K_{r}^{2} v}{V_{0}^{2}}
\end{aligned}
$$

Where $\mathrm{n}$ is a non-negative integer,

$\mathrm{U}_{0}, \mathrm{~T}_{0}$ are respectively the free stream velocity and mean temperature.

equations (2) and (7) dimensionless,

We introduce the following transformation:

$$
\frac{\partial u}{\partial t}-\left(1+\varepsilon e^{n^{\prime} t^{\prime}}\right) \frac{\partial u}{\partial y}=G_{r} \theta+G_{m} \varphi+\frac{\partial^{2} u}{\partial y^{2}}-\left(M+\frac{1}{K}\right) u
$$

Using equations (8), (9), and (11) the equations (2) and (7) are becomes

$$
\begin{gathered}
\frac{\partial \theta}{\partial t}-\left(1+\varepsilon e n^{\prime} t^{\prime}\right) \frac{\partial \theta}{\partial y}=\frac{1}{P_{r}}\left(1+\frac{4}{3 R}\right) \frac{\partial^{2} \theta}{\partial y^{2}}+E_{c}\left(\frac{\partial u}{\partial y}\right)^{2} \\
\frac{\partial \varphi}{\partial t}-\left(1+\varepsilon e^{n^{\prime} t^{\prime}}\right) \frac{\partial \varphi}{\partial y}=\frac{1}{S_{c}} \frac{\partial^{2} \varphi}{\partial y^{2}}-K_{r}^{2} \varphi
\end{gathered}
$$

Where $G_{r}=\frac{g_{0} \beta\left(T_{0}-T_{\infty}\right) \delta_{0}^{2}}{v U_{0}}$ is the local Grashof number, $M=\frac{\sigma B_{0}^{2} \delta^{2}}{\rho v}$ is the local magnetic parameter, $\operatorname{Pr}=\frac{\mathrm{v}}{\alpha}$ is the Prandtl number, $D a=\frac{k}{\delta^{2}}$ is the local Darcy number, $\operatorname{Re}=\frac{v_{0} \delta}{v}$ is the local Reynolds number, $F s=\frac{b}{\delta}$ is the Forchhemier number and $F s_{1}=\frac{b}{\delta}\left(\frac{\delta}{\delta_{0}}\right)^{2 n+2}$ Re is the modified Forchhemier number, $N=\frac{k k_{1}}{4 \sigma_{1} T_{\infty}^{3}}$ is the radiation number. 
The corresponding boundary conditions for $\mathrm{t}>0$ are given by

$$
\begin{aligned}
& \mathrm{u}=1, \theta=1+\varepsilon \mathrm{e}^{\mathrm{nt}}, \varphi=1+\varepsilon \mathrm{e}^{\mathrm{nt}} \text { at } \mathrm{y}=0 \\
& \mathrm{u} \rightarrow \mathrm{U}(\mathrm{t}), \theta \rightarrow 0, \varphi \rightarrow 0 \text { at } \mathrm{y} \rightarrow \infty
\end{aligned}
$$

\section{Results And Discussion}

1. For the purpose of discussing the results, the numerical calculations are presented in the form of nondimensional velocity and temperature profiles. Numerical computations have been carried out for different values of the parameters entering into the problem. The values of Grashof number (Gr) are taken to be large from the physical point of view.

2. The large Grashof number values correspond to free convection problem. The effects of suction parameter $\mathrm{v}_{0}$ on the velocity and temperature profiles .

3. we found that the velocity decreases with the increase of suction for cooling of the plate and increases for the heating of the plate. It is also clear that suction stabilizes the boundary layer growth. reveals that temperature decreases with the increase of the suction parameter.

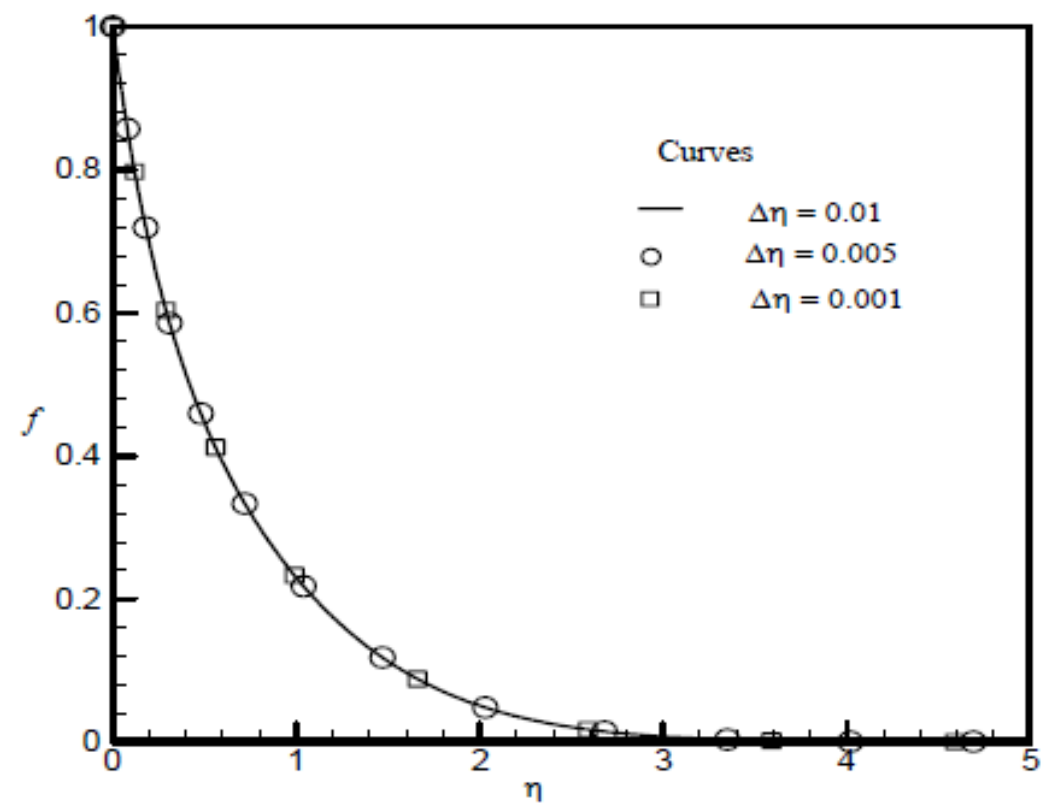

Fig. 1: Velocity profiles for different step sizes

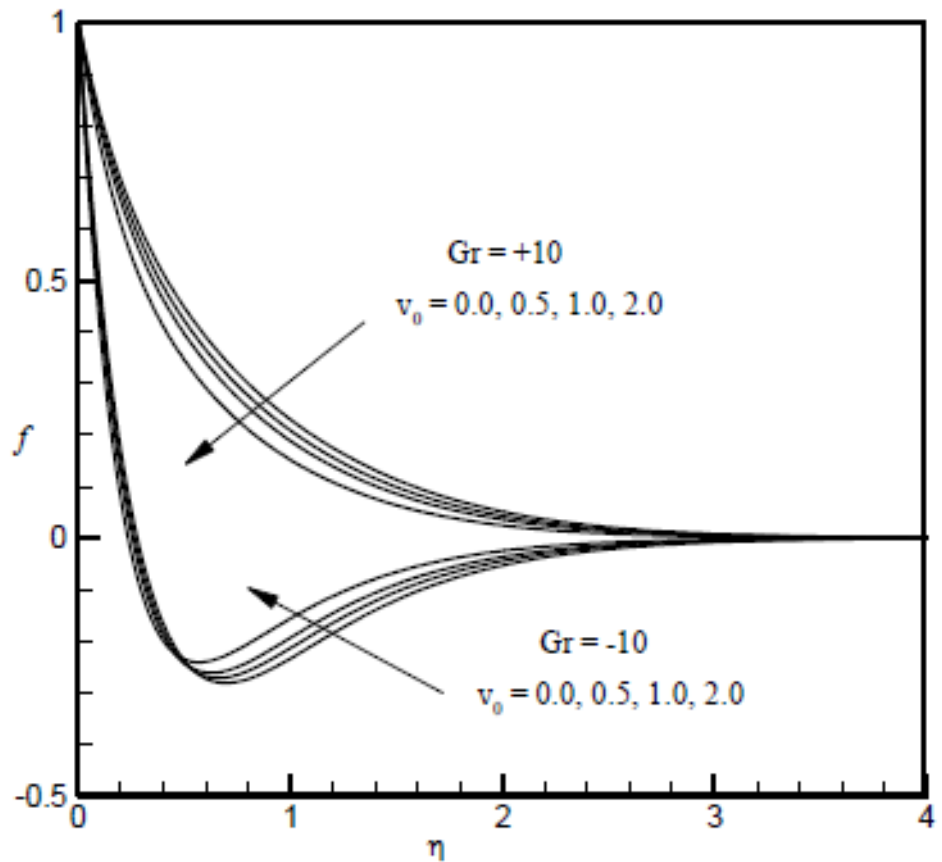

Fig. 2: Velocity profiles for different values of suction parameter $\left(\mathrm{v}_{0}\right)$ 


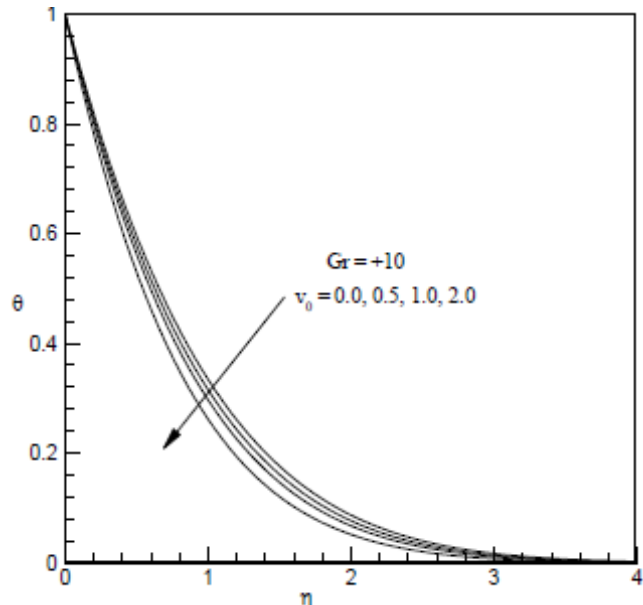

Fig 3.

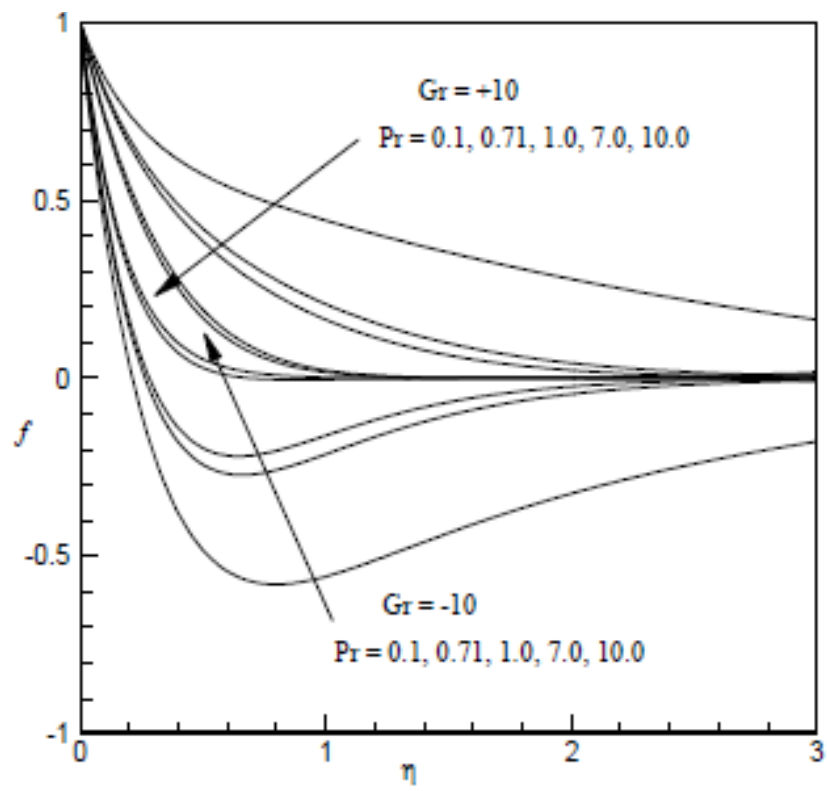

fig;4

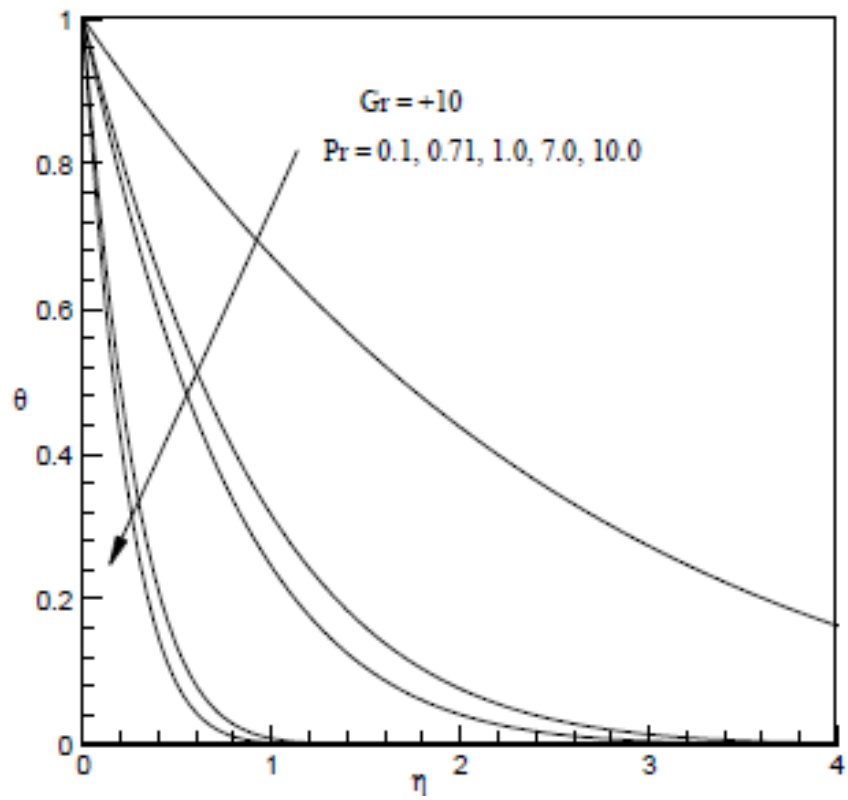

Fig:5 
Fig. 5 show the effects of Prandtl number (Pr) on the velocity as well as temperature profiles for cooling plate velocity profiles decrease with the increase of Pr whereas these profiles increase with the increase of Pr for a heating plate. For cooling plate Pr has decreasing effect on the temperature profiles.

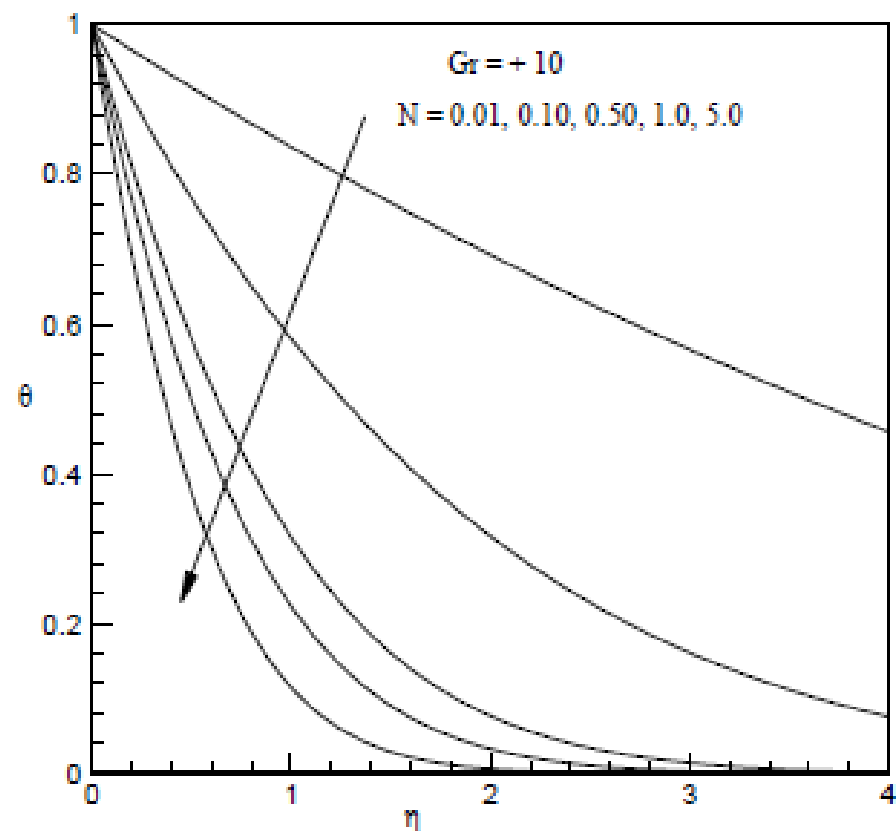

Fig. 6: Temperature profiles for different values of radiation parameter $(\mathrm{N})$

\section{Conclusion}

The thermal radiation communication with unsteady MHD frontier layer flow precedent a endlessly moving vertical porous plate absorbed in a porous medium. From the present study we can make the following conclusions:

(i) The suction stabilizes the boundary layer growth.

(ii) The velocity profiles increase whereas temperature profiles decrease with an increase of the free convection currents.

(iii) Using magnetic field we can control the flow characteristics and heat transfer.

(iv) Radiation has significant effects on the velocity as well as temperature distributions.

(v) Flow characteristics strongly depend on the nonnegative integer $n$.

(vi) Large Darcy number leads to the increase of the velocity profiles.

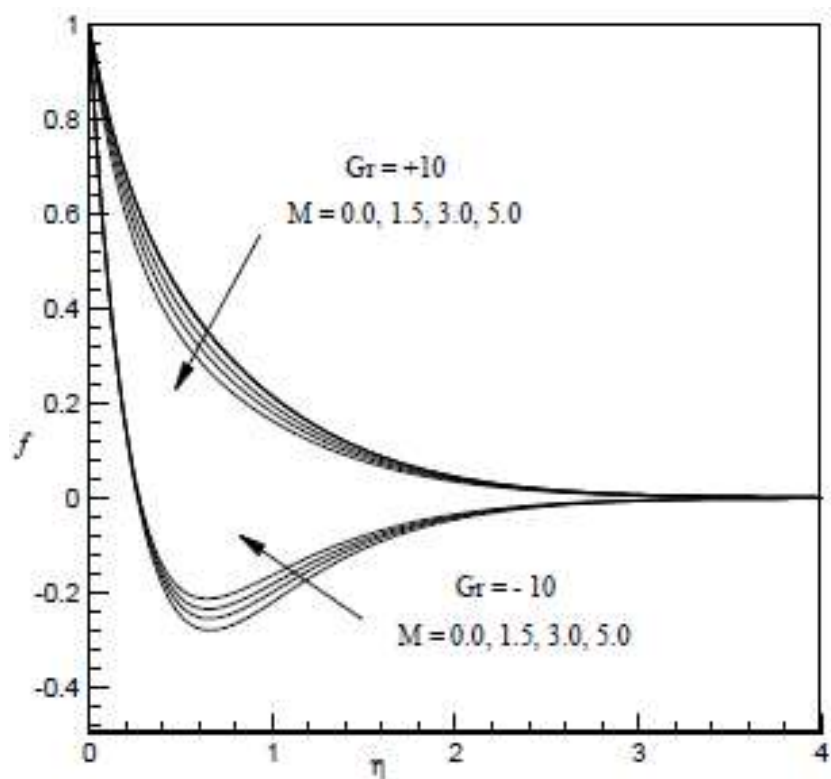

Fig. 7: Velocity Profiles for values of Magnetic parameter (M) 


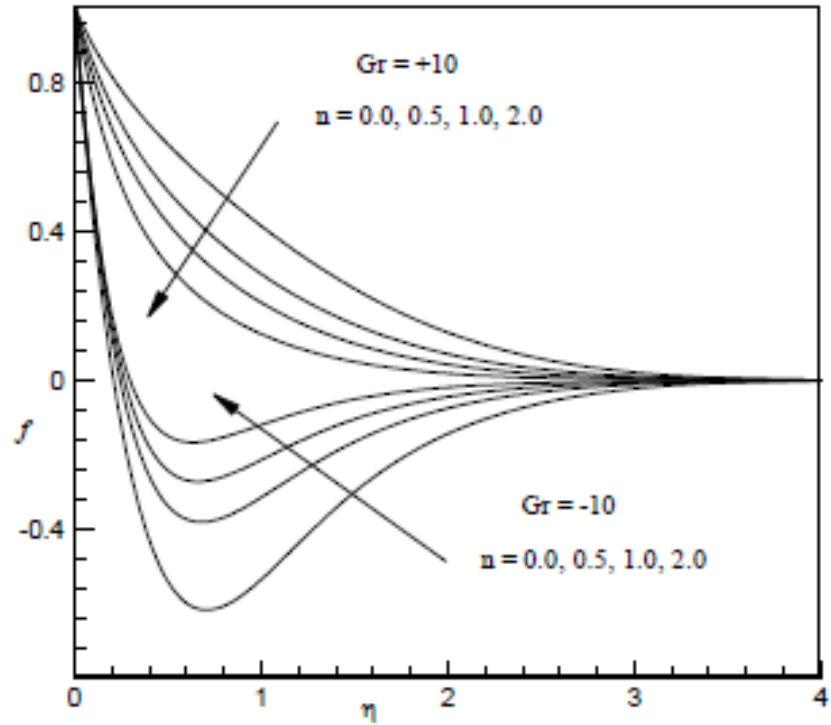

Fig. 8: Velocity profiles for different values nonnegative integer (n)

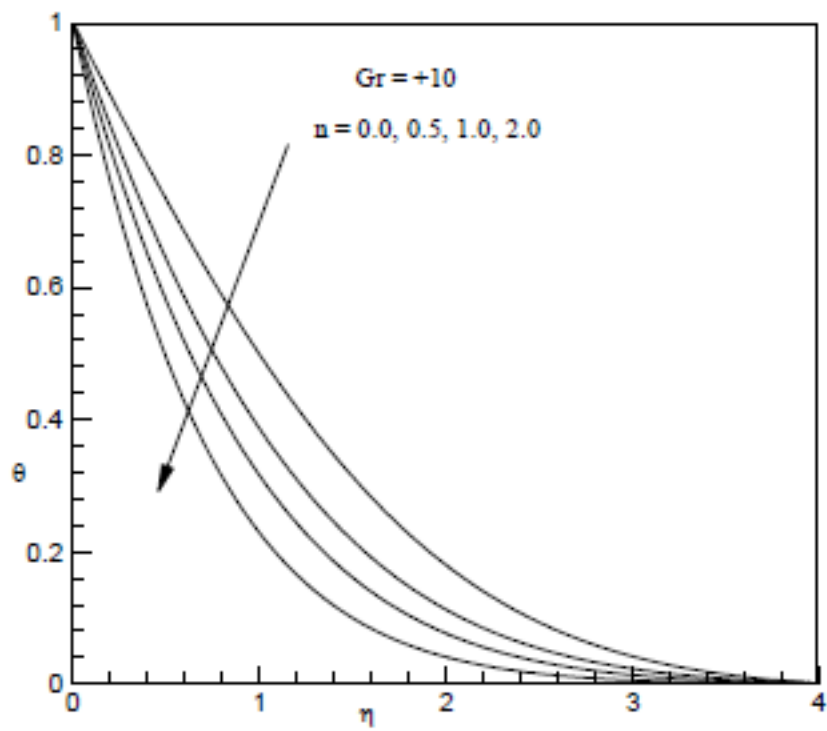

Fig. 9: Temperature profiles for different Values of non-negative integer (n)

\section{References}

[1] Alabraba, M. A., Bestman, A. R. and Ogulu, A. (1992): Laminar Convection in Binary Mixer of Hydromagnetic Flow with Radiative Heat Transfer. Astophys. Sapce Sci., Vol.195, pp. 431-445.

[2] Alam, M. S. and Rahman, M. M. (2005): Dufour and Soret Effects on MHD Free Convective Heat and Mass Transfer Flow Past a Vertical Porous Flat Plate Embedded in a Porous Medium. J. Nav. Arc. Mar. Eng., Vol.2(1), pp.55-65.

[3] Ali, M. M., Chen, T. S. and Armaly, B. F. ( 1984): Natural Convection Radiation Interaction in Boundary Layer Flow Over Horizontal Surfaces. AIAA Journal., Vol.22(12), pp. 797-1803.

[4] El-Arabawy, H. A. M. (2003): Effect of Suction/Injection on a Micropolar Fluid Past a Continuously Moving Plate in the Presence of Radiation. Intl. J. Heat Mass Trans., vol.46, pp.1471-1477.

[5] Ferdows, M., Sattar, M. A. and Siddiki, M. N. A. (2004): Numerical Approach on Parameters of the Thermal Radiation Interaction with Convection in a Boundary Layer Flow at a Vertical Plate with Variable Suction. Thammasat Intl. J. Sci. Tech., Vol.9(3), pp.19-28.

[6] Hashimoto, H. (1957): Boundary Layer Growth on a Flat Plate with Suction or Injection, J. Phys. Soc. Japan, Vol.22, pp.7-21.

[7] Hossain, M. A. and Takhar, H. S. (1996): Radiation Effect on Mixed Convection Along a Vertical Plate with Uniform Surface Temperature. Heat and Mass Trans., Vol.31(4), pp.243-248.

[8] Mansour, M. A. (1990): Radiative and Free Convection Effects on the Oscillatory Flow Past a Vertical Plate. Astophys. Sapce Sci., Vol.166, pp.269-275.

[9] Nachtsheim, P. R. and Swigert, P. (1965): Satisfaction of the Asymptotic Boundary Conditions in Numerical Solution of the System of Non-linear Equations of Boundary Layer Type, NASSA TND-3004.

[10] Raptis, A. (1983): Unsteady Free Convection Flow Through a Porous Medium. Intl. J. Eng. Sci., vol.21(4), pp.345-349.

[11] Raptis, A. and Perdikis, P. C. (1985): Unsteady Flow Through a Porous Medium in the Presence of Free Convection. Intl. Comm. Heat Mass Trans., Vol.12, pp.697-704. 
[12] Sattar, M. A. (1992): Free and Forced Convection Flow Through a Porous Medium Near the Leading Edge. Astrophys. Space Sci., Vol.191, pp.323-328.

[13] Sattar, M. A., and Hossain, M. M. (1992): Unsteady Hydromagnetic Free Convection Flow with Hall Current and Mass Transfer along an Accelerated Porous Plate with Time Dependent Temperature and Concentration, Can. J. Phys., Vol.70, pp.369-375.

[14] Sattar, M. A. and Kalim, H. (1996): Unsteady Free Convection Interaction with Thermal Radiation in a Boundary Layer Flow Past a Vertical Porous Plate. J. Math. Phys. Sci., vol.30(1), pp.25-37.

[15] Sattar, M. A. and Maleque, M. A. (2000): Unsteady MHD natural convection flow along an accelerated porous plate with hall current and mass transfer in a rotating porous medium, J. Energy, Heat and Mass Transfer., Vol.22, pp.67-72.

[16] Sattar, M. A., Rahman, M. M. and Alam, M. M. (2000): Free Convection Flow and Heat Transfer Through a Porous Vertical Flat Plate Immersed in a Porous Medium. J. Energy Res., Vol.22(1), pp.17-21.Siegel, R. (1958): Trans. Amer. Soc. Mech. Eng., Vol.80, pp.347.

[17] Singh, A. K. and Dikshit, C. K. (1988): Hydromagnetic Flow Past a Continuously Moving Semi-Infinite Plate for Large Suction. Astrophys. Space Sci., Vol.148, pp.249-256.

[18] Soundalgekar, V. M. and Takhar, H. S. (1981): MHD Flow Past a Vertical Oscillating Plate. Nucl. Eng. Des., Vol.64(1), pp.43-48.

[19] Soundalgekar, V. M., Vighnesam, N. V. and Pop, I. (1981): Combined Free and Forced Convection Flow Past a Vertical Porous Plate. Intl. J. Energy Res., Vol.5, pp.215-226.

[20] Yamamoto, K. and Iwamura, N. (1976): Flow with Convection Acceleration Through a Porous Medium. J. Eng. Math., Vol.10(1), pp.41-54.

\section{Nomenclature}

$\mathrm{c}_{\mathrm{f}} \quad$ skin resistance coefficient

$\mathrm{c}_{\mathrm{p}} \quad$ specific heat at constant pressure

Da local Darcy number

Ec Eckert number

Fs local Forchhemier number

$\mathrm{Fs}_{1} \quad$ modified Forchhemier number

$\mathrm{Gr}$ local Grashof number

g acceleration due to gravity

M local magnetic field parameter

$\mathrm{N}$ radiation parameter

$\mathrm{Nu} \quad$ Nusselt number

$\mathrm{n} \quad$ nonnegative integer

Pr Prandtl number

$\mathrm{q}_{\mathrm{r}} \quad$ radiative heat flux

$\mathrm{Re} \quad$ local Reynolds number

$\mathrm{T}$ temperature within the boundary layer

$\mathrm{T}(\mathrm{t}) \quad$ temperature at the plate

$\mathrm{T}_{\infty} \quad$ temperature of the ambient fluid

$\mathrm{t}$ time

$\mathrm{u} \quad$ velocity along $\mathrm{x}$-axis

$\mathrm{v} \quad$ velocity along $\mathrm{y}$-axis

$\mathrm{v}_{0} \quad$ suction parameter

$\mathrm{v}_{0}(\mathrm{t})$ time dependent suction velocity

$\mathrm{x} \quad$ coordinate along the plate

$\mathrm{y}$ coordinate normal to the plate 